\title{
Leech Method of Blood Analysis; A New Micro Method.
}

\section{Determination of Blood Sodium.*}

\author{
By \\ Yoshitaka Uga. \\ (宇贺義䓔)
}

(From the Pediatric Department, Faculty of Medicine, Tohoku

Imperial University, Sendai. Director: Prof. A. Sato.)

The very convenient leech method for the blood analysis of cholesterol $^{1)}$ and potassium ${ }^{23}$ has already been reported from our pediatric laboratory. Now I want to study the applicability of the leech method to sodium determination.

\section{The Leech Method for Blood Sodium.}

The colorimetrical blood sodium method of Yoshimatsu ${ }^{3)}$ was used. Table I gives a comparison of figures between blood samples obtained directly from a rabbit's vein, and those obtained through punction from leeches' bodies. Normal rabbits were used. The average deviation is within $\pm 3.3 \%$.

A comparison of sodium figures found in the blood of pathological human cases between the direct and the indirect (leech) method is shown in Table II.

As will be seen from Table II the figures obtained by the direct and the indirect method coincide, also in human cases, with each other within an average difference of $\pm 3 \%$.

\section{Conclusions.}

Using the leech method above described blood sodium may be de-

* 17th Report on Micro Method.

1) T. Sato, Tohoku Journ. Exp. Med., 1932, 19, 162.

2) Y. Ug a, Tohoku Journ. Exp. Med., 1932, $19,165$.

3) S. Yoshimats u, Tohoku Journ. Exp. Med., 1927, 8, 496. 
termined indirectly. The figures for blood sodium between the direct method and the indirect (leech) method coincide with each other within an average deviation of $\pm 3.3 \%$.

\section{TABLE I.}

Analysis of sodium in blood samples directly (from auricular vein) and indirectly through leech's body obtained from the same rabbits.

\begin{tabular}{|c|c|c|c|c|}
\hline $\begin{array}{l}\text { No. of } \\
\text { rabbits }\end{array}$ & $\begin{array}{l}\text { Body weight } \\
\text { in kilos }\end{array}$ & \begin{tabular}{|} 
Sodinm figures of \\
normal rabbit's \\
blood obtained \\
directly from its \\
auricular vein, \\
Na...mgrms $\%$
\end{tabular} & \begin{tabular}{|} 
Sodium figures of \\
the same rabbit's \\
blood obtained \\
indirectly from \\
the body of leech. \\
Na...mgrms $\%$
\end{tabular} & $\begin{array}{c}\text { Difference } \\
(\%)\end{array}$ \\
\hline $\begin{array}{lr}\text { No. } 1 \\
\text { No. } 2 \\
\text { No. } 3 \\
\text { No. } 4 \\
\text { No. } 5 \\
\text { No. } 6 \\
\text { No. } 7 \\
\text { No. } 8 \\
\text { No. } 9 \\
\text { No. } 10 \\
\text { No. } 11 \\
\text { No. } 12 \\
\text { No. } 13 \\
\text { No. } 14 \\
\text { No. } 15 \\
\text { No. } 16 \\
\text { No. } 17 \\
\text { No. } 18 \\
\text { No. } 19 \\
\text { No. } 20\end{array}$ & $\begin{array}{l}1.90 \\
1.77 \\
2.15 \\
2.32 \\
2.08 \\
2.64 \\
2.02 \\
1.79 \\
2.54 \\
1.99 \\
2.80 \\
2.24 \\
2.80 \\
2.07 \\
1.81 \\
2.69 \\
2.23 \\
2.05 \\
2.07 \\
1.95\end{array}$ & $\begin{array}{l}190.5 \\
222 \\
180 \\
191.5 \\
156 \\
206.5 \\
183 \\
202.5 \\
249 \\
227 \\
222 \\
180 \\
176.5 \\
237.5 \\
194 \\
163 \\
156.5 \\
236 \\
153 \\
180.5\end{array}$ & $\begin{array}{l}191 \\
218.5 \\
174 \\
181.5 \\
152 \\
204.5 \\
192 \\
208 \\
241 \\
214.5 \\
217 \\
192 \\
167 \\
226 \\
194.5 \\
157 \\
161 \\
235.5 \\
153.5 \\
165 \\
\text { A verage }\end{array}$ & $\begin{array}{l}+0.3 \\
-2.1 \\
-3.4 \\
-5.2 \\
-2.6 \\
-1.0 \\
+4.9 \\
+3.2 \\
-3.2 \\
-5.5 \\
-2.3 \\
+6.7 \\
-5.4 \\
-4.8 \\
+0.3 \\
-3.7 \\
-2.9 \\
-0.2 \\
+0.3 \\
-8.6 \\
\pm 3.3\end{array}$ \\
\hline
\end{tabular}

TABLE II.

Sodium content of blood in human cases (Na...mgrms. \%).

\begin{tabular}{|c|c|c|c|c|c|c|}
\hline No. & $\left|\begin{array}{c}\text { Age } \\
\text { (years) }\end{array}\right|$ & Sex & Diagnosis & $\begin{array}{c}\text { Blood obtained } \\
\text { directly }\end{array}$ & $\begin{array}{c}\text { Blood obtained } \\
\text { indirectly from } \\
\text { the body of } \\
\text { leech }\end{array}$ & Difference \\
\hline 1 & 15 & $\delta$ & $\begin{array}{l}\text { Polyserositis tuber- } \\
\text { culosa }\end{array}$ & 171 mgrms $\%$ & 179 mgrms $\%$ & $+4.7 \%$ \\
\hline 2 & 13 & 3 & Brain tumor & 162 mgrms $\%$ & $163 \mathrm{mgrms} \%$ & $+0.6 \%$ \\
\hline 3 & 17 & $\hat{\delta}$ & $\begin{array}{l}\text { Distrophia adiposo- } \\
\text { genitalis }\end{array}$ & $147 \mathrm{mgrms} \%$ & 152 mgrms $\%$ & $+3.4 \%$ \\
\hline 4 & 12 & 우 & $\begin{array}{l}\text { Hepatolienal disease } \\
\text { with jaundice }\end{array}$ & $187 \mathrm{mgrms} \%$ & 179 mgrms \% & $-4.3 \%$ \\
\hline 5 & 11 & $\hat{\delta}$ & Hepatitis luetica & 198 mgrms $\%$ & $\begin{array}{c}195 \text { mgrms } \% \\
\text { A verage }\end{array}$ & $\begin{array}{l}-1.5 \% \\
\pm 2.9 \%\end{array}$ \\
\hline
\end{tabular}

